\title{
HYBRID SPLINE FRAMES
}

\author{
SAY SONG GOH, TIM N. T. GOODMAN, AND S. L. LEE
}

\begin{abstract}
Using their unitary extension principle, Ron and Shen have constructed a normalized tight frame for $L^{2}(\mathbb{R})$ consisting of spline functions with uniform knots. This paper constructs a normalized tight frame for $L^{2}((0, \infty))$ comprising spline functions with knots on a hybrid of uniform and geometric mesh. The construction is motivated by applications in adaptive approximation using spline functions on a hybrid mesh that admits a natural dyadic multiresolution approximation of $L^{2}((0, \infty))$ based on dilation and translation.
\end{abstract}

\section{INTRODUCTION}

Cardinal splines or polynomial splines on $\mathbb{R}$ with integer knots were introduced and studied by Schoenberg in a series of papers (see for instance [20, 21]). They have been used by various authors (for instance [6, 7, 10, 11, 19]) to construct wavelet bases and frames for $L^{2}(\mathbb{R})$. In particular, Chui and Wang 10 constructed the semi-orthogonal spline wavelets and Ron and Shen [19] constructed normalized tight frames of spline functions for $L^{2}(\mathbb{R})$. Polynomial splines on geometric mesh, $q^{i}, i \in \mathbb{Z}$, or geometric splines were first studied by Micchelli [18]. They were independently rediscovered by Mallat in the study of image foveation [17. In this paper we consider spline functions on $(0, \infty)$ with knots on a hybrid of geometric and uniform meshes. More precisely, take an integer $n \geq 2$, and let $\widetilde{V}_{0}$ denote the space of all spline functions of order $n$ in $L^{2}((0, \infty))$ with $\operatorname{knots} x_{i}, i \in \mathbb{Z}$, where

$$
x_{i}= \begin{cases}2^{i}, & i \leq-1, \\ i+1, & i \geq 0 .\end{cases}
$$

The knot sequence, $x_{i}, i \in \mathbb{Z}$, is a hybrid of uniform and geometric meshes, and the corresponding piecewise polynomial functions will be referred to as hybrid uniformgeometric splines or simply hybrid splines. For $j \in \mathbb{Z}$, let $\widetilde{V}_{j}:=\left\{f\left(2^{j} \cdot\right): f \in \widetilde{V}_{0}\right\}$. For each $j, \widetilde{V}_{j}$ is the space of polynomial splines of order $n$ on $(0, \infty)$ with knots at $2^{-j} x_{i}, i \in \mathbb{Z}$, where $x_{i}$ are as in (1.1). We shall construct a normalized tight frame for $L^{2}((0, \infty))$ of compactly supported functions in $\widetilde{V}_{j}, j \in \mathbb{Z}$.

This construction, which is motivated by applications in adaptive approximation, such as image foveation ([3, 4, 17]) and feature preserving smoothing [12, is a follow up of the construction in 12 of Riesz bases of wavelets for $L^{2}((0, \infty))$ from

Received by the editor September 26, 2006 and, in revised form, March 15, 2008.

2000 Mathematics Subject Classification. Primary 65D07, 41A15; Secondary 42C40, 42 C30.

Key words and phrases. Uniform splines, geometric splines, hybrid splines, multiresolution, tight frames.

This research was partially supported by the Wavelets and Information Processing Programme of the Centre for Wavelets, Approximation and Information Processing, National University of Singapore, under a grant from DSTA.

(C)2009 American Mathematical Society 1537 
functions in $\widetilde{V}_{j}, j \in \mathbb{Z}$. It was shown in [12] that unlike the case of uniform splines, there is no Riesz basis of compactly supported hybrid spline wavelets for $L^{2}((0, \infty))$ for $n \geq 2$, which are orthogonal across different levels of scaling. One of the objectives of this paper is to overcome this shortcoming by considering tight frames instead of Riesz bases. It turns out that tight frames of compactly supported hybrid splines exist for $L^{2}((0, \infty))$ and their construction is more natural and more elegant and the analysis is much simpler than those of the Riesz bases. They will be more versatile in applications, which may have wider ramifications, because the processes of foveation, smoothing and compression are not restricted to images but are applicable to a wider class of objects, including those in vision research, 3-D visualization and virtual reality $([1,2,3,15])$, and in the multiresolution processing of geometry information ([14, [16]).

The multiresolution setting of hybrid splines and our construction of the corresponding tight frames are different from those studied by Chui, He and Stöckler in [8, 9]. In 9] they developed a theory together and a general construction of nonstationary tight frames for $L^{2}(I)$, where $I=[0, \infty)$ or $\mathbb{R}$, using the kernels of certain approximation operators on the multiresolution subspaces. In particular, they studied in greater detail multiresolution subspaces of spline functions with arbitrary nested knot sequences on $\mathbb{R}$ and on the semi-infinite interval $[0, \infty)$, where 0 is considered a multiple knot with maximum multiplicity. However, explicit construction of nonstationary tight frames for specific cases remains an interesting and challenging problem, even in the special case of cubic spline frames derived from the general non-stationary construction in [9].

Our approach in the construction of tight frames of hybrid splines uses a direct method based on matrix extension in the time domain. The idea is to integrate uniform splines on a half infinite interval with geometric splines near the origin. It is possible to extend the idea to develop a theory and general method of construction of tight frames of spline functions for $L^{2}(0, \infty)$, with arbitrary nested knot sequences, but the explicit construction of tight frames for specific cases will remain a technically difficult problem. The salient point in the case of hybrid splines is the simplicity and elegance of the multiresolution structure, the construction and the results, which hold for arbitrary degree. Interestingly, the hybrid spline tight frames (Theorem 3.1) comprise the dyadic dilations of a finite set of compactly supported spline functions with a mix of uniform and geometric knots and a single geometric $B$-spline with knots $2^{\ell}, \ell=0,1, \ldots, n$, together with the dyadic dilations and shifts by positive integers of the uniform spline frame generators of Ron and Shen [19]. In Section 2 we construct a normalized tight frame of uniform splines for $L^{2}([0, \infty))$ from the nonnegative integer shifts of the uniform spline frame generators and the uniform $B$-spline with knots at $0,1, \ldots, n$. They are then used in Section 3 for the construction of tight frames of hybrid splines for $L^{2}((0, \infty))$.

\section{SPline FRAMES ON THE HALF LiNE}

Take an integer $n \geq 2$. Let $\mathcal{S}_{n}$ denote the space of all spline functions of order $n$, i.e. $C^{n-2}$ piecewise polynomials of degree $n-1$, with knots in $\mathbb{Z}$. Let

$$
V_{0}:=\left\{f \in \mathcal{S}_{n}: \operatorname{supp}(f) \subset[0, \infty)\right\} \cap L^{2}(\mathbb{R})
$$

and

$$
V_{j}:=\left\{f\left(2^{j} \cdot\right): f \in V_{0}\right\}, \quad j \in \mathbb{Z}
$$


We shall construct a normalized tight frame for $L^{2}([0, \infty))$ from functions in $V_{j}$, $j \in \mathbb{Z}$, where for $f \in V_{j}$ we confuse $f$ and $\left.f\right|_{[0, \infty)}$.

Let $N \in \mathcal{S}_{n}$ denote the $B$-spline with support $[0, n]$, normalized by $\int_{0}^{n} N=1$, so that $\sum_{j=-\infty}^{\infty} N(\cdot-j)=1$. Note that

$$
N(x)=\sum_{i=0}^{n} a_{0 i}^{n} N(2 x-i), \quad x \in \mathbb{R},
$$

where

$$
P_{0}^{n}(z):=2^{-n+1}(1+z)^{n}=: \sum_{i=0}^{n} a_{0 i}^{n} z^{i} .
$$

For $k=1,2, \ldots, n$, let

$$
P_{k}^{n}(z):=2^{-n+1}(1-z)^{k}(1+z)^{n-k}=: \sum_{i=0}^{n} a_{k i}^{n} z^{i}
$$

and

$$
\psi_{k}(x):=\sqrt{\left(\begin{array}{l}
n \\
k
\end{array}\right)} \sum_{i=0}^{n} a_{k i}^{n} N(2 x-i), \quad x \in \mathbb{R} .
$$

By applying the 'unitary extension principle', introduced by Ron and Shen in [19, it can easily be seen that the functions

$$
2^{j / 2} \psi_{k}\left(2^{j} \cdot-i\right), i, j \in \mathbb{Z}, k=1,2, \ldots, n,
$$

form a normalized tight frame for $L^{2}(\mathbb{R})$. The essential idea of this construction was given in [19]. We shall see that the functions $\psi_{k}, k=1,2, \ldots, n$, in (2.3) together with the uniform $B$-spline will lead to a normalized tight frame for $L^{2}([0, \infty))$.

Theorem 2.1. Let $S \subset L^{2}([0, \infty))$ denote the set

$$
\psi_{k}(\cdot-i), i=0,1, \ldots, k=1,2, \ldots, n, c_{\ell}^{1 / 2} N(2 \cdot-\ell), \ell=0,1, \ldots, n-2,
$$

where

$$
c_{\ell}:=2^{-n+1} \sum_{j=\ell+1}^{n-1}\left(\begin{array}{c}
n-1 \\
j
\end{array}\right), \quad \ell=0,1, \ldots, n-2 .
$$

Then $\left\{2^{j / 2} f\left(2^{j} \cdot\right): f \in S, j \in \mathbb{Z}\right\}$ is a normalized tight frame for $L^{2}([0, \infty))$.

We shall need the following lemma to prove the theorem.

Lemma 2.2. For $n \geq 1$ and $i, j \in\{0,1, \ldots, n\}$,

$$
\sum_{k=0}^{n}\left(\begin{array}{l}
n \\
k
\end{array}\right) a_{k i}^{n} a_{k j}^{n}= \begin{cases}0, & i \neq j, \\
2^{-n+2}\left(\begin{array}{c}
n \\
i
\end{array}\right), & i=j .\end{cases}
$$

Proof. We prove by induction on $n$. The identity is easily seen to be true for $n=1$. Assume it is true for some $n \geq 1$. Now for $k=0,1, \ldots, n$,

$$
P_{k}^{n+1}(z)=2^{-1}(1+z) P_{k}^{n}(z), \quad P_{k+1}^{n+1}(z)=2^{-1}(1-z) P_{k}^{n}(z),
$$

and hence for $i=0,1, \ldots, n+1$,

$$
a_{k i}^{n+1}=\frac{1}{2}\left(a_{k, i-1}^{n}+a_{k i}^{n}\right), \quad a_{k+1, i}^{n+1}=\frac{1}{2}\left(a_{k i}^{n}-a_{k, i-1}^{n}\right),
$$


where we have taken $a_{k,-1}^{n}=a_{k, n+1}^{n}=0$. Then for $i, j \in\{0,1, \ldots, n+1\}$,

$$
\begin{aligned}
\sum_{k=0}^{n+1}\left(\begin{array}{c}
n+1 \\
k
\end{array}\right) a_{k i}^{n+1} a_{k j}^{n+1}= & \sum_{k=0}^{n+1}\left(\left(\begin{array}{c}
n \\
k-1
\end{array}\right)+\left(\begin{array}{l}
n \\
k
\end{array}\right)\right) a_{k i}^{n+1} a_{k j}^{n+1} \\
= & \sum_{k=0}^{n}\left(\begin{array}{l}
n \\
k
\end{array}\right) \frac{1}{4}\left(a_{k i}^{n}-a_{k, i-1}^{n}\right)\left(a_{k j}^{n}-a_{k, j-1}^{n}\right) \\
& +\sum_{k=0}^{n}\left(\begin{array}{l}
n \\
k
\end{array}\right) \frac{1}{4}\left(a_{k, i-1}^{n}+a_{k i}^{n}\right)\left(a_{k, j-1}^{n}+a_{k j}^{n}\right) \\
= & \frac{1}{2} \sum_{k=0}^{n}\left(\begin{array}{l}
n \\
k
\end{array}\right)\left(a_{k, i-1}^{n} a_{k, j-1}^{n}+a_{k i}^{n} a_{k j}^{n}\right) .
\end{aligned}
$$

So our induction hypothesis gives for $i \neq j$,

$$
\sum_{k=0}^{n+1}\left(\begin{array}{c}
n+1 \\
j
\end{array}\right) a_{k i}^{n+1} a_{k j}^{n+1}=0
$$

and for $i=j$,

$$
\begin{aligned}
\sum_{k=0}^{n+1}\left(\begin{array}{c}
n+1 \\
k
\end{array}\right)\left(a_{k i}^{n+1}\right)^{2} & =\frac{1}{2} \sum_{k=0}^{n}\left(\begin{array}{l}
n \\
k
\end{array}\right)\left(a_{k, i-1}^{n}\right)^{2}+\frac{1}{2} \sum_{k=0}^{n}\left(\begin{array}{l}
n \\
k
\end{array}\right)\left(a_{k i}^{n}\right)^{2} \\
& =2^{-n+1}\left(\begin{array}{c}
n \\
i-1
\end{array}\right)+2^{-n+1}\left(\begin{array}{c}
n \\
i
\end{array}\right)=2^{-n+1}\left(\begin{array}{c}
n+1 \\
i
\end{array}\right) .
\end{aligned}
$$

Thus, the result is true for $n+1$, and this concludes the inductive proof.

Whether (2.4) forms a normalized tight frame depends on the following (see [5], p. 319). For simplicity we write $\langle f, g\rangle:=\int_{-\infty}^{\infty} f g$ for $f, g \in L^{2}(\mathbb{R})$, and for $j \in \mathbb{Z}$, $N_{j}:=N(\cdot-j), \widetilde{N}_{j}:=\sqrt{2} N(2 \cdot-j), \psi_{k j}:=\psi_{k}(\cdot-j), k=1,2, \ldots, n$. Then for $f \in L^{2}(\mathbb{R})$,

$$
\sum_{j=-\infty}^{\infty}\left\langle f, \widetilde{N}_{j}\right\rangle^{2}=\sum_{j=-\infty}^{\infty}\left\langle f, N_{j}\right\rangle^{2}+\sum_{j=-\infty}^{\infty} \sum_{k=1}^{n}\left\langle f, \psi_{k j}\right\rangle^{2} .
$$

(In fact, in [5] this is stated for $f$ in a dense subset of $L^{2}(\mathbb{R})$, but by passing to the limit it easily follows that it holds for all $L^{2}(\mathbb{R})$.)

In a similar manner Theorem 2.1 is based upon the following lemma, where $\langle f, g\rangle$ now denotes $\int_{0}^{\infty} f g$ for $f, g \in L^{2}([0, \infty))$.

Lemma 2.3. For $f \in L^{2}([0, \infty))$,

$$
\sum_{j=0}^{\infty}\left\langle f, \widetilde{N}_{j}\right\rangle^{2}=\sum_{j=0}^{\infty}\left\langle f, N_{j}\right\rangle^{2}+\sum_{j=0}^{\infty} \sum_{k=1}^{n}\left\langle f, \psi_{k j}\right\rangle^{2}+\sum_{j=0}^{n-2} c_{j}\left\langle f, \widetilde{N}_{j}\right\rangle^{2}
$$

Proof. Take $f \in L^{2}([0, \infty))$. By (2.6),

$$
\sum_{j=1-n}^{\infty}\left\langle f, \widetilde{N}_{j}\right\rangle^{2}=\sum_{j=1-n}^{\infty}\left\langle f, N_{j}\right\rangle^{2}+\sum_{j=1-n}^{\infty} \sum_{k=1}^{n}\left\langle f, \psi_{k j}\right\rangle^{2} .
$$


Thus (2.7) is equivalent to

$$
\sum_{j=1-n}^{-1}\left\langle f, N_{j}\right\rangle^{2}+\sum_{j=1-n}^{-1} \sum_{k=1}^{n}\left\langle f, \psi_{k j}\right\rangle^{2}-\sum_{j=1-n}^{-1}\left\langle f, \widetilde{N}_{j}\right\rangle^{2}=\sum_{j=0}^{n-2} c_{j}\left\langle f, \widetilde{N}_{j}\right\rangle^{2},
$$

which is

$$
\frac{1}{2} \sum_{j=1-n}^{-1} \sum_{k=0}^{n}\left(\begin{array}{l}
n \\
k
\end{array}\right)\left\langle f, \sum_{i=0}^{n} a_{k i}^{n} \widetilde{N}_{i+2 j}\right\rangle^{2}-\sum_{j=1-n}^{-1}\left\langle f, \widetilde{N}_{j}\right\rangle^{2}=\sum_{j=0}^{n-2} c_{j}\left\langle f, \widetilde{N}_{j}\right\rangle^{2},
$$

by (2.1) and (2.3). Letting $\alpha_{j}:=\left\langle f, \widetilde{N}_{j}\right\rangle, j \in \mathbb{Z}$, (2.8) becomes

$$
\frac{1}{2} \sum_{j=1-n}^{-1} \sum_{k=0}^{n}\left(\begin{array}{l}
n \\
k
\end{array}\right)\left(\sum_{i=0}^{n} a_{k i}^{n} \alpha_{i+2 j}\right)^{2}-\sum_{j=1-n}^{-1} \alpha_{j}^{2}=\sum_{j=0}^{n-2} c_{j} \alpha_{j}^{2} .
$$

Now applying Lemma 2.2,

$$
\begin{aligned}
\frac{1}{2} \sum_{j=1-n}^{-1} \sum_{k=0}^{n}\left(\begin{array}{l}
n \\
k
\end{array}\right)\left(\sum_{i=0}^{n} a_{k i}^{n} \alpha_{i+2 j}\right)^{2} & =\frac{1}{2} \sum_{j=1-n}^{-1} \sum_{i=0}^{n} \sum_{\ell=0}^{n} \alpha_{i+2 j} \alpha_{\ell+2 j} \sum_{k=0}^{n}\left(\begin{array}{l}
n \\
k
\end{array}\right) a_{k i}^{n} a_{k \ell}^{n} \\
& =2^{-n+1} \sum_{j=1-n}^{-1} \sum_{i=0}^{n}\left(\begin{array}{c}
n \\
i
\end{array}\right) \alpha_{i+2 j}^{2} .
\end{aligned}
$$

Performing further calculations and recalling $\alpha_{j}=0, j=2-2 n, \ldots,-n$, we have

$$
\begin{aligned}
2^{-n+1} \sum_{j=1-n}^{-1} \sum_{i=0}^{n}\left(\begin{array}{c}
n \\
i
\end{array}\right) \alpha_{i+2 j}^{2} & =2^{-n+1} \sum_{j=1-n}^{-1} \sum_{i=0}^{n}\left\{\left(\begin{array}{c}
n-1 \\
i-1
\end{array}\right)+\left(\begin{array}{c}
n-1 \\
i
\end{array}\right)\right\} \alpha_{i+2 j}^{2} \\
& =2^{-n+1} \sum_{\ell=0}^{n-1}\left(\begin{array}{c}
n-1 \\
\ell
\end{array}\right) \sum_{j=\ell+2-2 n}^{\ell-1} \alpha_{j}^{2} \\
& =2^{-n+1} \sum_{j=1-n}^{n-2} \alpha_{j}^{2} \sum_{\ell=j+1}^{n-1}\left(\begin{array}{c}
n-1 \\
\ell
\end{array}\right) \\
& =\sum_{j=1-n}^{-1} \alpha_{j}^{2}+\sum_{j=0}^{n-2} c_{j} \alpha_{j}^{2} .
\end{aligned}
$$

Hence by (2.10) and (2.11), we obtain (2.9) which gives (2.7) and completes the proof.

Proof of Theorem 2.1. Take $f \in L^{2}([0, \infty))$. By scaling (2.7) we see that for any $j \in \mathbb{Z}$

$$
\begin{aligned}
\sum_{i=0}^{\infty}\left\langle f, 2^{(j+1) / 2} N\left(2^{j+1} \cdot-i\right)\right\rangle^{2}=\sum_{i=0}^{\infty}\left\langle f, 2^{j / 2} N\left(2^{j} \cdot-i\right)\right\rangle^{2} \\
\quad+\sum_{i=0}^{\infty} \sum_{k=1}^{n}\left\langle f, 2^{j / 2} \psi_{k}\left(2^{j} \cdot-i\right)\right\rangle^{2}+\sum_{i=0}^{n-2} c_{i}\left\langle f, 2^{(j+1) / 2} N\left(2^{j+1} \cdot-i\right)\right\rangle^{2} .
\end{aligned}
$$


So for any integers $r<s$,

$$
\begin{aligned}
\sum_{i=0}^{\infty}\left\langle f, 2^{s / 2} N\left(2^{s} \cdot-i\right)\right\rangle^{2}=\sum_{i=0}^{\infty}\left\langle f, 2^{r / 2} N\left(2^{r} \cdot-i\right)\right\rangle^{2} \\
\quad+\sum_{j=r}^{s-1} \sum_{i=0}^{\infty} \sum_{k=1}^{n}\left\langle f, 2^{j / 2} \psi_{k}\left(2^{j} \cdot-i\right)\right\rangle^{2}+\sum_{j=r+1}^{s} \sum_{i=0}^{n-2} c_{i}\left\langle f, 2^{j / 2} N\left(2^{j} \cdot-i\right)\right\rangle^{2} .
\end{aligned}
$$

It is well known that

$$
\lim _{s \rightarrow \infty} \sum_{i=-\infty}^{\infty}\left\langle f, 2^{s / 2} N\left(2^{s} \cdot-i\right)\right\rangle 2^{s / 2} N\left(2^{s} \cdot-i\right)=f
$$

in $L^{2}([0, \infty))$. (For more general results, see [13].) It follows immediately that

$$
\lim _{s \rightarrow \infty} \sum_{i=-\infty}^{\infty}\left\langle f, 2^{s / 2} N\left(2^{s} \cdot-i\right)\right\rangle^{2}=\|f\|^{2} .
$$

Taking any $\epsilon>0$ and applying (2.13) to $\left.f\right|_{[0, \epsilon)}$, we see that

$$
\varlimsup_{s \rightarrow \infty} \sum_{i=1-n}^{-1}\left\langle f, 2^{s / 2} N\left(2^{s} \cdot-i\right)\right\rangle^{2} \leq \int_{0}^{\epsilon} f^{2}
$$

and letting $\epsilon \rightarrow 0$ shows that

$$
\lim _{s \rightarrow \infty} \sum_{i=1-n}^{-1}\left\langle f, 2^{s / 2} N\left(2^{s} \cdot-i\right)\right\rangle^{2}=0 .
$$

Since $\left\langle f, N\left(2^{s} \cdot-i\right)\right\rangle=0$ for $i \leq-n,(2.13)$ and (2.14) give

$$
\lim _{s \rightarrow \infty} \sum_{i=0}^{\infty}\left\langle f, 2^{s / 2} N\left(2^{s} \cdot-i\right)\right\rangle^{2}=\|f\|^{2} .
$$

It is shown in $([5$, p. 320) that

$$
\lim _{r \rightarrow-\infty} \sum_{i=-\infty}^{\infty}\left\langle f, 2^{r / 2} N\left(2^{r} \cdot-i\right)\right\rangle^{2}=0
$$

and hence

$$
\lim _{r \rightarrow-\infty} \sum_{i=0}^{\infty}\left\langle f, 2^{r / 2} N\left(2^{r} \cdot-i\right)\right\rangle^{2}=0 .
$$

From (2.12), (2.15) and (2.16) we then have

$$
\|f\|^{2}=\sum_{j=-\infty}^{\infty} \sum_{i=0}^{\infty} \sum_{k=1}^{n}\left\langle f, 2^{j / 2} \psi_{k}\left(2^{j} \cdot-i\right)\right\rangle^{2}+\sum_{j=-\infty}^{\infty} \sum_{i=0}^{n-2} c_{i}\left\langle f, 2^{j / 2} N\left(2^{j} \cdot-i\right)\right\rangle^{2},
$$

which proves the result. 


\section{SPline Frames on A HYBRID Mesh}

Again take an integer $n \geq 2$, the knot sequence $x_{i}, i \in \mathbb{Z}$, as in (1.1) and as before let $\widetilde{V}_{0}$ denote the space of all spline functions of order $n$ in $L^{2}((0, \infty))$ with knots $x_{i}, i \in \mathbb{Z}$, and $\widetilde{V}_{j}:=\left\{f\left(2^{j} \cdot\right): f \in \widetilde{V}_{0}\right\}$ for $j \in \mathbb{Z}$. We now construct a normalized tight frame for $L^{2}((0, \infty))$ from functions in $\widetilde{V}_{j}, j \in \mathbb{Z}$.

For $j \in \mathbb{Z}$, let $N_{j}$ denote the $B$-spline with knots $x_{j}, \ldots, x_{j+n}$, normalized so that

$$
\sum_{j=-\infty}^{\infty} N_{j}(x)=1, \quad x>0 .
$$

We write

$$
I_{j}:=\int_{0}^{\infty} N_{j}=\frac{x_{j+n}-x_{j}}{n}, \quad j \in \mathbb{Z} .
$$

Since $\widetilde{V}_{0} \subset \widetilde{V}_{1}$, the functions $\left(N_{j}\right)$ satisfy a refinement equation of the form

$$
N_{j}=\sum_{k=-\infty}^{\infty} b_{j k}^{n} N_{k}(2 \cdot), \quad j \in \mathbb{Z}
$$

For $j \geq 0, N_{j}=N(\cdot-j-1), I_{j}=1$, and from (2.1) and (2.2),

$$
N_{j}=2^{-n+1} \sum_{k=0}^{n}\left(\begin{array}{l}
n \\
k
\end{array}\right) N_{k+1+2 j}(2 \cdot) \text {. }
$$

Thus,

$$
b_{j k}^{n}=2^{-n+1}\left(\begin{array}{c}
n \\
k-2 j-1
\end{array}\right), \quad j \geq 0, k \in \mathbb{Z} .
$$

Note that for $j \leq 1-n, N_{j}$ has knots $2^{j}, \ldots, 2^{j+n}$, and so

$$
N_{j}=M\left(2^{-j} \cdot\right), \quad j \leq 1-n,
$$

where $M$ is the 'geometric' $B$-spline with knots $2^{\ell}, \ell=0,1, \ldots, n$, with $\int_{0}^{\infty} M=$ $\frac{2^{n}-1}{n}$. Thus,

$$
N_{j}=N_{j+1}(2 \cdot), \quad j \leq-n
$$

and

$$
b_{j k}^{n}=\delta_{j, k-1}, \quad j \leq-n .
$$

It can be shown that $b_{j k}^{n} \geq 0, j, k \in \mathbb{Z}$, and $b_{j k}^{n}>0$ if and only if

$$
\begin{cases}k=j+1, & j \leq-n, \\ j+1 \leq k \leq 2 j+n+1, & -n<j \leq 0, \\ 2 j+1 \leq k \leq 2 j+n+1, & j>0 .\end{cases}
$$

From (3.1) and (3.3),

$$
1=\sum_{j=-\infty}^{\infty} N_{j}=\sum_{k=-\infty}^{\infty} N_{k}(2 \cdot) \sum_{j=-\infty}^{\infty} b_{j k}^{n},
$$

and so

$$
\sum_{j=-\infty}^{\infty} b_{j k}^{n}=1, \quad k \in \mathbb{Z} .
$$


In order to state our main result, we define for integers $k, \ell$,

$$
c_{k \ell}:=\frac{1}{2} I_{k} I_{\ell} \sum_{j=1-n}^{-1} \frac{1}{I_{j}} b_{j k}^{n} b_{j \ell}^{n} .
$$

Clearly, $c_{k \ell}=c_{\ell k} \geq 0$ for any integers $k \neq \ell$. We can see from (3.7) that for $k<\ell$, $c_{k \ell}>0$ if and only if

$$
2-n \leq k<\ell \leq \min \{n-1+2 k, n-1\} .
$$

Note that (3.3) gives

$$
I_{j}=\frac{1}{2} \sum_{k=-\infty}^{\infty} b_{j k}^{n} I_{k}
$$

Theorem 3.1. Let $T \subset L^{2}((0, \infty))$ denote the set of functions $\left(\frac{n}{2^{n}-1}\right)^{\frac{1}{2}} M$, $\psi_{k}(\cdot-i), i=1,2, \ldots, k=1,2, \ldots, n$, and

$$
c_{\ell m}^{1 / 2}\left(\frac{N_{\ell}}{I_{\ell}}-\frac{N_{m}}{I_{m}}\right), \quad 2-n \leq \ell<m \leq \min \{n-1+2 \ell, n-1\} .
$$

Then $\left\{2^{j / 2} f\left(2^{j}.\right): f \in T, j \in \mathbb{Z}\right\}$ is a normalized tight frame for $L^{2}((0, \infty))$.

We shall see in due course that the $(n-1)^{2}$ functions in (3.11) can in fact be replaced by $2 n-3$ functions, but not of such a simple form. The form of (3.11) comes from the following.

Lemma 3.2. Let $A=\left(A_{i j}\right)_{i, j=1}^{m}$ be a symmetric matrix satisfying $\sum_{j=1}^{m} A_{i j}=0$, $i=1,2, \ldots, m$. Then for any $\beta_{1}, \ldots, \beta_{m}$,

$$
\sum_{i=1}^{m} \sum_{j=1}^{m} A_{i j} \beta_{i} \beta_{j}=-\sum_{1 \leq i<j \leq m} A_{i j}\left(\beta_{i}-\beta_{j}\right)^{2} .
$$

Proof. For any $\beta_{1}, \ldots, \beta_{m}$,

$$
\begin{aligned}
\sum_{1 \leq i<j \leq m} A_{i j}\left(\beta_{i}-\beta_{j}\right)^{2} & =\sum_{i=1}^{m} \beta_{i}^{2} \sum_{\substack{j=1 \\
j \neq i}}^{m} A_{i j}-2 \sum_{1 \leq i<j \leq m} A_{i j} \beta_{i} \beta_{j} \\
& =-\sum_{i=1}^{m} \beta_{i}^{2} A_{i i}-2 \sum_{1 \leq i<j \leq m} A_{i j} \beta_{i} \beta_{j} \\
& =-\sum_{i=1}^{m} \sum_{j=1}^{m} A_{i j} \beta_{i} \beta_{j} .
\end{aligned}
$$

Now, in a similar manner to Lemma 2.3, the proof of Theorem 3.1 is based on the following.

Lemma 3.3. For $f \in L^{2}((0, \infty))$,

$$
\begin{aligned}
2 \sum_{j=-\infty}^{\infty} I_{j}^{-1}\left\langle f, N_{j}(2 \cdot)\right\rangle^{2}= & \sum_{j=-\infty}^{\infty} I_{j}^{-1}\left\langle f, N_{j}\right\rangle^{2}+\sum_{j=1}^{\infty} \sum_{k=1}^{n}\left\langle f, \psi_{k j}\right\rangle^{2} \\
& +2 \sum_{2-n \leq \ell<m \leq n-1} c_{\ell m}\left\langle f, \frac{N_{\ell}(2 \cdot)}{I_{\ell}}-\frac{N_{m}(2 \cdot)}{I_{m}}\right\rangle^{2} .
\end{aligned}
$$


Proof. By Lemma 2.3 applied on $[1, \infty)$,

$$
2 \sum_{j=1}^{\infty}\left\langle f, N_{j}(2 \cdot)\right\rangle^{2}=\sum_{j=0}^{\infty}\left\langle f, N_{j}\right\rangle^{2}+\sum_{j=1}^{\infty} \sum_{k=1}^{n}\left\langle f, \psi_{k j}\right\rangle^{2}+2 \sum_{j=1}^{n-1} c_{j-1}\left\langle f, N_{j}(2 \cdot)\right\rangle^{2}
$$

Now let

$$
Y:=2 \sum_{j=-\infty}^{\infty} I_{j}^{-1}\left\langle f, N_{j}(2 \cdot)\right\rangle^{2}-\sum_{j=-\infty}^{\infty} I_{j}^{-1}\left\langle f, N_{j}\right\rangle^{2}-\sum_{j=1}^{\infty} \sum_{k=1}^{n}\left\langle f, \psi_{k j}\right\rangle^{2} .
$$

By (3.6) $, I_{j}^{-1} N_{j}=2 I_{j+1}^{-1} N_{j+1}(2 \cdot), j \leq-n$, and so

$$
Y=2 \sum_{j=2-n}^{\infty} I_{j}^{-1}\left\langle f, N_{j}(2 \cdot)\right\rangle^{2}-\sum_{j=1-n}^{\infty} I_{j}^{-1}\left\langle f, N_{j}\right\rangle^{2}-\sum_{j=1}^{\infty} \sum_{k=1}^{n}\left\langle f, \psi_{k j}\right\rangle^{2} .
$$

Adding (3.13) and (3.15) and recalling, $I_{j}=1, j \geq 0$, gives

$$
Y=2 \sum_{j=2-n}^{0} I_{j}^{-1}\left\langle f, N_{j}(2 \cdot)\right\rangle^{2}+2 \sum_{j=1}^{n-1} c_{j-1}\left\langle f, N_{j}(2 \cdot)\right\rangle^{2}-\sum_{j=1-n}^{-1} I_{j}^{-1}\left\langle f, N_{j}\right\rangle^{2} .
$$

Putting $\beta_{j}:=\sqrt{2} I_{j}^{-1}\left\langle f, N_{j}(2 \cdot)\right\rangle, j \in \mathbb{Z}$, and applying (3.3), this becomes

$$
Y=\sum_{j=2-n}^{0} I_{j} \beta_{j}^{2}+\sum_{j=1}^{n-1} c_{j-1} \beta_{j}^{2}-\sum_{j=1-n}^{-1} \frac{1}{2 I_{j}}\left\{\sum_{k=-\infty}^{\infty} b_{j k}^{n} I_{k} \beta_{k}\right\}^{2},
$$

which simplifies to

$$
Y=\sum_{j=2-n}^{0} I_{j} \beta_{j}^{2}+\sum_{j=1}^{n-1} c_{j-1} \beta_{j}^{2}-\sum_{k=2-n}^{n-1} \sum_{\ell=2-n}^{n-1} c_{k \ell} \beta_{k} \beta_{\ell},
$$

using (3.7) and (3.9). We may write this as

$$
Y=\sum_{k=2-n}^{n-1} \sum_{\ell=2-n}^{n-1} A_{k \ell} \beta_{k} \beta_{\ell}
$$

where $A=\left(A_{k \ell}\right)_{k, \ell=2-n}^{n-1}$ is a symmetric matrix given by

$$
\begin{cases}A_{k k}=I_{k}-c_{k k}, & k=2-n, \ldots, 0, \\ A_{k k}=c_{k-1}-c_{k k}, & k=1, \ldots, n-1, \\ A_{k \ell}=-c_{k \ell}, & 2-n \leq k<\ell \leq n-1 .\end{cases}
$$

We shall show that for $k=2-n, \ldots, n-1$,

$$
\sum_{\ell=2-n}^{n-1} A_{k \ell}=0 \text {. }
$$

First take $2-n \leq k \leq 0$. Then using (3.17) and (3.9),

$$
\begin{aligned}
\sum_{\ell=2-n}^{n-1} A_{k \ell} & =I_{k}-\frac{1}{2} I_{k} \sum_{j=1-n}^{-1} \frac{1}{I_{j}} b_{j k}^{n} \sum_{\ell=2-n}^{n-1} b_{j \ell}^{n} I_{\ell} \\
& =I_{k}-I_{k} \sum_{j=1-n}^{-1} b_{j k}^{n},
\end{aligned}
$$


by (3.10), on noting from (3.7) that $b_{j \ell}^{n}=0$ except for $2-n \leq j+1 \leq \ell \leq 2 j+n+1 \leq$ $n-1$. Also from (3.7) we see that $b_{j k}^{n}=0$ except for $1-n \leq \frac{k-n-1}{2} \leq j \leq k-1 \leq-1$. Thus

$$
\sum_{j=1-n}^{-1} b_{j k}^{n}=\sum_{j=-\infty}^{\infty} b_{j k}^{n}=1,
$$

by (3.8), and so (3.19) gives (3.18) .

Now take $1 \leq k \leq n-1$. As in the derivation of (3.19), and noting that $I_{k}=1$, we have

$$
\sum_{\ell=2-n}^{n-1} A_{k \ell}=c_{k-1}-\sum_{j=1-n}^{-1} b_{j k}^{n} .
$$

From (3.7) we see that $b_{j k}^{n} \neq 0$ implies $j \geq \frac{k-n-1}{2}$, and since $1-n \leq \frac{k-n-1}{2}$,

$$
\sum_{j=1-n}^{-1} b_{j k}^{n}=\sum_{j=-\infty}^{-1} b_{j k}^{n}=1-\sum_{j=0}^{\infty} b_{j k}^{n},
$$

by (3.8). Thus by (3.20), (2.5) and (3.4), we have

$$
\begin{aligned}
\sum_{\ell=2-n}^{n-1} A_{k \ell} & =2^{-n+1} \sum_{j=k}^{n-1}\left(\begin{array}{c}
n-1 \\
j
\end{array}\right)+2^{-n+1} \sum_{j=0}^{\infty}\left(\begin{array}{c}
n \\
k-2 j-1
\end{array}\right)-1 \\
& =2^{-n+1} \sum_{j=0}^{\infty}\left(\begin{array}{l}
n-1 \\
k+j
\end{array}\right)+2^{-n+1} \sum_{j=0}^{\infty}\left\{\left(\begin{array}{c}
n-1 \\
k-2 j-2
\end{array}\right)+\left(\begin{array}{c}
n-1 \\
k-2 j-1
\end{array}\right)\right\}-1 \\
& =2^{-n+1} \sum_{j=-\infty}^{\infty}\left(\begin{array}{l}
n-1 \\
k+j
\end{array}\right)-1 \\
& =2^{-n+1} 2^{n-1}-1=0 .
\end{aligned}
$$

Hence (3.18) holds for $k=2-n, \ldots, n-1$.

By Lemma 3.2, (3.16) and (3.17),

$$
\begin{aligned}
Y & =-\sum_{2-n \leq i<j \leq n-1} A_{i j}\left(\beta_{i}-\beta_{j}\right)^{2} \\
& =2 \sum_{2-n \leq i<j \leq n-1} c_{i j}\left\langle f, \frac{N_{i}(2 \cdot)}{I_{i}}-\frac{N_{j}(2 \cdot)}{I_{j}}\right\rangle^{2} .
\end{aligned}
$$

From (3.14), this gives (3.12).

Proof of Theorem 3.1. Take $f \in L^{2}((0, \infty))$. By scaling (3.12) we see that for any $j \in \mathbb{Z}$

$$
\begin{aligned}
& \sum_{i=-\infty}^{\infty} 2^{j+1} I_{i}^{-1}\left\langle f, N_{i}\left(2^{j+1} \cdot\right)\right\rangle^{2}=\sum_{i=-\infty}^{\infty} 2^{j} I_{i}^{-1}\left\langle f, N_{i}\left(2^{j} \cdot\right)\right\rangle^{2} \\
& \quad+\sum_{i=1}^{\infty} \sum_{k=1}^{n} 2^{j}\left\langle f, \psi_{k i}\left(2^{j} \cdot\right)\right\rangle^{2}+\sum_{2-n \leq \ell<m \leq n-1} 2^{j+1} c_{\ell m}\left\langle f, \frac{N_{\ell}\left(2^{j+1} \cdot\right)}{I_{\ell}}-\frac{N_{m}\left(2^{j+1} \cdot\right)}{I_{m}}\right\rangle^{2} .
\end{aligned}
$$


So for any integers $r<s$, we have

$$
\begin{aligned}
& \sum_{i=-\infty}^{\infty} I_{i}^{-1}\left\langle f, 2^{s / 2} N_{i}\left(2^{s} \cdot\right)\right\rangle^{2}=\sum_{i=-\infty}^{\infty} I_{i}^{-1}\left\langle f, 2^{r / 2} N_{i}\left(2^{r} \cdot\right)\right\rangle^{2} \\
& \quad+\sum_{j=r}^{s-1} \sum_{i=1}^{\infty} \sum_{k=1}^{n}\left\langle f, 2^{j / 2} \psi_{k i}\left(2^{j} \cdot\right)\right\rangle^{2} \\
& \quad+\sum_{j=r+1}^{s} \sum_{2-n \leq \ell<m \leq n-1} c_{\ell m}\left\langle f, 2^{j / 2}\left(\frac{N_{\ell}\left(2^{j} \cdot\right)}{I_{\ell}}-\frac{N_{m}\left(2^{j} \cdot\right)}{I_{m}}\right)\right\rangle^{2} .
\end{aligned}
$$

Now for any integers $i, s$, recalling from (3.1) that $0 \leq N_{i}(x) \leq 1, x>0$, we have

$$
\begin{aligned}
I_{i}^{-1}\left\langle f, 2^{\frac{s}{2}} N_{i}\left(2^{s} \cdot\right)\right\rangle^{2} & \leq 2^{s} I_{i}^{-1} \int_{2^{-s} x_{i}}^{2^{-s} x_{i+n}} f^{2} \int_{2^{-s} x_{i}}^{2^{-s} x_{i+n}} N_{i}\left(2^{s} \cdot\right)^{2} \\
& \leq I_{i}^{-1}\left(x_{i+n}-x_{i}\right) \int_{2^{-s} x_{i}}^{2^{-s} x_{i+n}} f^{2}=n \int_{2^{-s} x_{i}}^{2^{-s} x_{i+n}} f^{2},
\end{aligned}
$$

by (3.2). Thus,

$$
\begin{aligned}
\sum_{i=-\infty}^{-1} I_{i}^{-1}\left\langle f, 2^{s / 2} N_{i}\left(2^{s} \cdot\right)\right\rangle^{2} & \leq n \sum_{i=-\infty}^{-1} \int_{2^{-s} x_{i}}^{2^{-s} x_{i+n}} f^{2} \\
& \leq n^{2} \int_{0}^{2^{-s} x_{n-1}} f^{2} \rightarrow 0 \text { as } s \rightarrow \infty
\end{aligned}
$$

Also,

$$
\begin{aligned}
\sum_{i=0}^{\infty} I_{i}^{-1}\left\langle f, 2^{s / 2} N_{i}\left(2^{s} \cdot\right)\right\rangle^{2} & =\sum_{i=1}^{\infty}\left\langle f, 2^{s / 2} N\left(2^{s} \cdot-i\right)\right\rangle^{2} \\
& \rightarrow\|f\|^{2} \text { as } s \rightarrow \infty
\end{aligned}
$$

as in the proof of Theorem 2.1. As above,

$$
\begin{aligned}
\sum_{i=2-n}^{\infty} I_{i}^{-1}\left\langle f, 2^{r / 2} N_{i}\left(2^{r} \cdot\right)\right\rangle^{2} & \leq n \sum_{i=2-n}^{\infty} \int_{2^{-r} x_{i}}^{2^{-r} x_{i+n}} f^{2} \\
& \leq n^{2} \int_{2^{-r} x_{2-n}}^{\infty} f^{2} \rightarrow 0 \text { as } r \rightarrow-\infty
\end{aligned}
$$

Also by (3.5),

$$
\begin{aligned}
\sum_{i=-\infty}^{1-n} I_{i}^{-1}\left\langle f, 2^{r / 2} N_{i}\left(2^{r} \cdot\right)\right\rangle^{2} & =\sum_{i=-\infty}^{1-n} \frac{2^{-i} n}{2^{n}-1}\left\langle f, 2^{r / 2} M\left(2^{r-i} \cdot\right)\right\rangle^{2} \\
& =\sum_{j=-\infty}^{1-n-r} \frac{n}{2^{n}-1}\left\langle f, 2^{-j / 2} M\left(2^{-j} \cdot\right)\right\rangle^{2}
\end{aligned}
$$


From (3.21)-3.251,

$$
\begin{aligned}
\|f\|^{2}= & \sum_{j=-\infty}^{\infty} \frac{n}{2^{n}-1}\left\langle f, 2^{-j / 2} M\left(2^{-j} \cdot\right)\right\rangle^{2}+\sum_{j=-\infty}^{\infty} \sum_{i=1}^{\infty} \sum_{k=1}^{r}\left\langle f, 2^{j / 2} \psi_{k i}\left(2^{j} \cdot\right)\right\rangle^{2} \\
& +\sum_{j=-\infty}^{\infty} \sum_{2-r \leq \ell<m \leq n-1} c_{\ell m}\left\langle f, 2^{j / 2}\left(\frac{N_{\ell}\left(2^{j} \cdot\right)}{I_{\ell}}-\frac{N_{m}\left(2^{j} \cdot\right)}{I_{m}}\right)\right\rangle^{2},
\end{aligned}
$$

which proves the result.

Now recall the matrix $A$, as in (3.17), and consider the Cholesky type factorization $A=L L^{T}$. Here $L=\left(L_{k \ell}\right)_{k, \ell=2-n}^{n-1}$, where $L_{k \ell}=0$ unless

$$
2-n \leq \ell \leq k \leq \min \{n-1+2 \ell, n-1\}
$$

and

$$
\sum_{k=\ell}^{n-1} L_{k \ell}=0, \quad 2-n \leq \ell \leq n-1
$$

Then $L_{n-1, n-1}=0$ and from (3.16),

$$
\begin{aligned}
Y & =\sum_{\ell=2-n}^{n-2}\left(\sum_{k=\ell}^{n-1} L_{k \ell} \beta_{k}\right)^{2} \\
& =2 \sum_{\ell=2-n}^{n-2}\left\langle f, \sum_{k=\ell}^{n-1} L_{k \ell} I_{k}^{-1} N_{k}(2 \cdot)\right\rangle^{2}
\end{aligned}
$$

So in Theorem 3.1 we may replace the $(n-1)^{2}$ functions in (3.11) by the $2 n-3$ functions

$$
\sum_{k=\ell}^{n-1} L_{k \ell} \frac{N_{k}}{I_{k}}, \quad \ell=2-n, \ldots, n-2
$$

Example. Take $n=2$. From (3.9), $c_{01}=\frac{1}{2 I_{-1}} b_{-1,0}^{2} b_{-1,1}^{2}$. Since $b_{-1,0}^{2}=1, b_{-1,1}^{2}=$ $\frac{1}{2}, I_{-1}=\frac{x_{1}-x_{-1}}{2}=\frac{3}{4}$, we have $c_{01}=\frac{1}{3}$, and so (3.11) comprises the function $\frac{1}{\sqrt{3}}\left(N_{0}-N_{1}\right)$.

Example. Take $n=3$. Here $b_{-1,0}^{3}=\frac{2}{3}$ and the other values follow from (3.4) or (3.8). From (3.9), the matrix $A$ in (3.17) is given by

$$
A=\left[\begin{array}{cccc}
\frac{5}{21} & -\frac{5}{21} & 0 & 0 \\
-\frac{5}{21} & \frac{67}{105} & -\frac{3}{10} & -\frac{1}{10} \\
0 & -\frac{3}{10} & \frac{33}{80} & -\frac{9}{80} \\
0 & -\frac{1}{10} & -\frac{9}{80} & \frac{17}{80}
\end{array}\right]
$$

Thus (3.11) comprises

$$
\frac{\sqrt{105}}{105}\left(6 N_{-1}-5 N_{0}\right), \frac{\sqrt{30}}{10}\left(N_{0}-N_{1}\right), \frac{\sqrt{10}}{10}\left(N_{0}-N_{2}\right), \frac{3 \sqrt{5}}{20}\left(N_{1}-N_{2}\right) .
$$


A simple calculation shows $A=L L^{T}$, where

$$
L=\left[\begin{array}{cccc}
\frac{\sqrt{105}}{21} & 0 & 0 & 0 \\
-\frac{\sqrt{105}}{21} & \frac{\sqrt{10}}{5} & 0 & 0 \\
0 & -\frac{3 \sqrt{10}}{20} & \frac{\sqrt{3}}{4} & 0 \\
0 & -\frac{\sqrt{10}}{20} & -\frac{\sqrt{3}}{4} & 0
\end{array}\right] .
$$

So we may replace (3.11) by the three functions:

$$
\frac{\sqrt{105}}{105}\left(6 N_{-1}-5 N_{0}\right), \frac{\sqrt{10}}{20}\left(4 N_{0}-3 N_{1}-N_{2}\right), \frac{\sqrt{3}}{4}\left(N_{1}-N_{2}\right) \text {. }
$$

Example. Take $n=4$. Here $\left(b_{j, k}^{4}\right)_{j=-4, k=-4}^{1,5}$ is

$$
\left[\begin{array}{cccccccccc}
0 & 1 & 0 & 0 & 0 & 0 & 0 & 0 & 0 & 0 \\
0 & 0 & 1 & \frac{2}{7} & 0 & 0 & 0 & 0 & 0 & 0 \\
0 & 0 & 0 & \frac{5}{7} & \frac{3}{5} & \frac{3}{20} & 0 & 0 & 0 & 0 \\
0 & 0 & 0 & 0 & \frac{2}{5} & \frac{29}{40} & \frac{1}{2} & \frac{1}{8} & 0 & 0 \\
0 & 0 & 0 & 0 & 0 & \frac{1}{8} & \frac{1}{2} & \frac{3}{4} & \frac{1}{2} & \frac{1}{8} \\
0 & 0 & 0 & 0 & 0 & 0 & 0 & \frac{1}{8} & \frac{1}{2} & \frac{3}{4}
\end{array}\right]
$$

From (3.9), the functions in (3.11) are

$$
\begin{aligned}
& \frac{4 \sqrt{165}}{1155}\left(14 N_{-2}-11 N_{-1}\right), \frac{\sqrt{33}}{77}\left(8 N_{-1}-7 N_{0}\right), \frac{\sqrt{33}}{154}\left(8 N_{-1}-7 N_{1}\right), \\
& \frac{\sqrt{34265}}{385}\left(N_{0}-N_{1}\right), \frac{2 \sqrt{35}}{35}\left(N_{0}-N_{2}\right), \frac{\sqrt{1015}}{70}\left(N_{1}-N_{2}\right), \\
& \frac{\sqrt{35}}{35}\left(N_{0}-N_{3}\right), \frac{\sqrt{1015}}{140}\left(N_{1}-N_{3}\right), \frac{\sqrt{7}}{14}\left(N_{2}-N_{3}\right) .
\end{aligned}
$$

Also from (3.9), the matrix $A$ in (3.17) is given by

$$
A=\left[\begin{array}{cccccc}
\frac{11}{60} & -\frac{11}{60} & 0 & 0 & 0 & 0 \\
-\frac{11}{60} & \frac{173}{330} & -\frac{3}{11} & -\frac{3}{44} & 0 & 0 \\
0 & -\frac{3}{11} & \frac{249}{385} & -\frac{89}{385} & -\frac{4}{35} & -\frac{1}{35} \\
0 & -\frac{3}{44} & -\frac{89}{385} & \frac{3439}{6160} & -\frac{29}{140} & -\frac{29}{560} \\
0 & 0 & -\frac{4}{35} & -\frac{29}{140} & \frac{5}{14} & -\frac{1}{28} \\
0 & 0 & -\frac{1}{35} & -\frac{29}{560} & -\frac{1}{28} & \frac{13}{112}
\end{array}\right] .
$$

A simple calculation shows $A=L L^{T}$, where

$$
L=\left[\begin{array}{cccccc}
\frac{\sqrt{165}}{30} & 0 & 0 & 0 & 0 & 0 \\
-\frac{\sqrt{165}}{30} & \frac{\sqrt{165}}{22} & 0 & 0 & 0 & 0 \\
0 & -\frac{2 \sqrt{165}}{55} & \frac{\sqrt{21}}{7} & 0 & 0 & 0 \\
0 & -\frac{\sqrt{165}}{110} & -\frac{2 \sqrt{21}}{21} & \frac{\sqrt{51}}{12} & 0 & 0 \\
0 & 0 & -\frac{4 \sqrt{21}}{105} & -\frac{\sqrt{51}}{15} & \frac{\sqrt{10}}{10} & 0 \\
0 & 0 & -\frac{\sqrt{21}}{105} & -\frac{\sqrt{51}}{60} & -\frac{\sqrt{10}}{10} & 0
\end{array}\right] .
$$


So we may replace (3.11) by the five functions:

$$
\begin{aligned}
& \frac{\sqrt{165}}{1155}\left(14 N_{-2}-11 N_{-1}\right), \frac{\sqrt{165}}{110}\left(40 N_{-1}-28 N_{0}-7 N_{1}\right), \\
& \frac{\sqrt{21}}{105}\left(15 N_{0}-10 N_{1}-4 N_{2}-N_{3}\right), \frac{\sqrt{51}}{60}\left(5 N_{1}-4 N_{2}-N_{3}\right), \frac{\sqrt{10}}{10}\left(N_{2}-N_{3}\right) .
\end{aligned}
$$

\section{REFERENCES}

[1] A. Basu, I. Cheng, Y. Pan, Foveated Online 3D Visualization, 16th International Conference on Pattern Recognition (ICPR'02), Canada, Volume 3, pp. 30 - 44.

[2] M. Bolduc and M. D. Levine, A Real-time Foveated Sensor with Overlapping Receptive Fields, Real-Time Imaging, 3(1977), 195 - 212.

[3] P. J. Burt, Smart sensing within a pyramid vision machine, Proc. IEEE, 76 (1988), 1006 1015.

[4] E. C. Chang, S. Mallat and C. Yap, Wavelet foveation, Appl. Comput. Harmon. Anal., 9 (2000), 312 - 335. MR.1793421 (2002g:94003)

[5] O. Christensen, An Introduction to Frames and Riesz Bases, Birkhaüser, Boston, 2003. MR1946982(2003k:42001)

[6] C. K. Chui and W. He, Compactly supported tight frames associated with refinable functions, Appl. Comput. Harmon. Anal., 8 (2000), 293 - 319. MR.1754930 (2001h:42049)

[7] C. K. Chui, W. He and J. Stöckler, Compactly supported tight and sibling frames with maximum vanishing moments, Appl. Comput. Harmon. Anal., 13 (2002), 224 - 262. MR1942743 (2004a:94011)

[8] C. K. Chui, W. He and J. Stöckler, Nonstationary tight wavelet frames, I: bounded intervals, Appl. Comput. Harmon. Anal., 17 (2004), 141 - 197. MR2082157 (2005f:42082)

[9] C. K. Chui, W. He and J. Stöckler, Nonstationary tight wavelet frames, II: unbounded intervals, Appl. Comput. Harmon. Anal., 18 (2005), 25 - 66. MR2110512 (2005j:42026)

[10] C. K. Chui and J. Z. Wang, On compactly supported spline wavelets and a duality principle, Trans. Amer. Math. Soc., 330 (1992), 903 - 915. MR.1076613 (92f:41020)

[11] I. Daubechies, B. Han, A. Ron and Z. Shen, Framelets: MRA-based constructions of wavelet frames, Appl. Comput. Harmon. Anal., 14 (2003), 1 - 46. MR1971300 (2004a:42046)

[12] X. Gao, T. N. T. Goodman and S. L. Lee, Foveated splines and wavelets, Appl. Comput. Harmon. Anal. (to appear)

[13] K. Jetter and D.-X. Zhou, Order of linear approximation from shift-invariant spaces, Constr. Approx., 11 (1995), 423 - 438. MR1367171(96i:41024)

[14] A. Khodakovsky, P. Schröder, W. Sweldens, Progressive geometry compression, in: Proc. ACM SIGGRAPH, 2000, pp. 271-278.

[15] Y. Kuniyoshi, K. Nobuyuki, S. Rougeaux, T, Suehiro, Active Stereo Vision System with Foveated Wide Angle Lens, Proceedings of 2nd Asian Conference on Computer Vision, Singapore, 1995, pp. $191-200$.

[16] M. Lounsbery, T.D. DeRose, J. Warren, Multiresolution analysis for surfaces of arbitrary topology type, ACM Trans. Graphics 16 (1997), 34-73.

[17] S. Mallat, Foveal detection and approximation for singularities, Appl. Comput. Harmon. Anal., 14 (2003), 133 - 180. MR1981205 (2004c:42072)

[18] C. A. Micchelli, Cardinal $\mathcal{L}$-splines, in Studies in Spline Functions and Approximation Theory, Academic Press, New York, 1976, pp. 203 - 250. MR0481767 (58:1866)

[19] A. Ron and Z. Shen, Affine systems in $L_{2}\left(\mathbb{R}^{d}\right)$ : the analysis of the analysis operator, J. Funct. Anal., 148 (1997), 408 - 447. MR.1469348 (99g:42043)

[20] I. J. Schoenberg, Cardinal interpolation and spline functions, J. Approx. Theory, 2 (1969), 167 - 206. MR0257616 (41:2266)

[21] I. J. Schoenberg, Cardinal Spline Interpolation, CBMS-NSF Regional Conference Series in Applied Mathematics 12, SIAM, 1973. MR0420078 (54:8095) 
Department of Mathematics, National University of Singapore, 10 Kent Ridge CresCEnt, Singapore 119260

E-mail address: matgohss@nus.edu.sg

Department of Mathematics, The University of Dundee, Dundee DD1 4hN, Scotland, UNITED KINGDOM

E-mail address: tgoodman@maths.dundee.ac.uk

Department of Mathematics, National University of Singapore, 10 Kent Ridge CresCEnT, Singapore 119260

E-mail address: matleesl@nus.edu.sg 